\title{
HAJEK-RENYI-TYPE INEQUALITY FOR SOME NONMONOTONIC FUNCTIONS OF ASSOCIATED RANDOM VARIABLES
}

\author{
ISHA DEWAN AND B. L. S. PRAKASA RAO
}

Received 21 April 2005; Revised 26 October 2005; Accepted 11 December 2005

Let $\left\{Y_{n}, n \geq 1\right\}$ be a sequence of nonmonotonic functions of associated random variables. We derive a Newman and Wright (1981) type of inequality for the maximum of partial sums of the sequence $\left\{Y_{n}, n \geq 1\right\}$ and a Hajek-Renyi-type inequality for nonmonotonic functions of associated random variables under some conditions. As an application, a strong law of large numbers is obtained for nonmonotonic functions of associated random varaibles.

Copyright (C) 2006 I. Dewan and B. L. S. P. Rao. This is an open access article distributed under the Creative Commons Attribution License, which permits unrestricted use, distribution, and reproduction in any medium, provided the original work is properly cited.

\section{Introduction}

Let $\{\Omega, \mathscr{F}, \mathscr{P}\}$ a probability space and $\left\{X_{n}, n \geq 1\right\}$ be a sequence of associated random variables defined on it. A finite collection $\left\{X_{1}, X_{2}, \ldots, X_{n}\right\}$ is said to be associated if for every pair of functions $h(\mathbf{x})$ and $g(\mathbf{x})$ from $\mathbb{R}^{n}$ to $\mathbb{R}$, which are nondecreasing componentwise,

$$
\operatorname{Cov}(h(\mathbf{X}), g(\mathbf{X})) \geq 0
$$

whenever it is finite, where $\mathbf{X}=\left(X_{1}, X_{2}, \ldots, X_{n}\right)$. The infinite sequence $\left\{X_{n}, n \geq 1\right\}$ is said to be associated if every finite subfamily is associated.

Associated random variables are of considerable interest in reliability studies (cf. Barlow and Proschan [1], Esary et al. [6]), statistical physics (cf. Newman [9, 10]), and percolation theory (cf. Cox and Grimmet [4]). For an extensive review of several probabilistic and statistical results for associated sequences, see Roussas [14] and Dewan and Rao [5].

Newman and Wright [12] proved an inequality for maximum of partial sums and Prakasa Rao [13] proved the Hajek-Renyi-type inequality for associated random variables. Esary et al. [6] proved that monotonic functions of associated random variables are associated. Hence one can easily extend the above-mentioned inequalities to monotonic 
functions of associated random variables. We now generalise the above results to some nonmonotonic functions of associated random variables.

In Section 2, we discuss some preliminaries. Two inequalities are proved for nonmonotonic functions of associated random variables in Section 3. As an application, a strong law of large numbers is derived for nonmonotonic functions of associated random variables in Section 4.

\section{Preliminaries}

Let us discuss some definitions and results which will be useful in proving our main results.

Definition 2.1 (Newman [11]). Let $f$ and $f_{1}$ be two real-valued functions defined on $\mathbb{R}^{n}$. Then $f \ll f_{1}$ if and only if $f_{1}+f$ and $f_{1}-f$ are both nondecreasing componentwise. In particular, if $f \ll f_{1}$, then $f_{1}$ will be nondecreasing componentwise.

Dewan and Rao [5] observed the following.

Remark 2.2. Suppose that $f$ is a real-valued function defined on $\mathbb{R}$. Then $f \ll f_{1}$ for some real-valued function defined $f_{1}$ on $\mathbb{R}$ if and only if for $x<y$,

$$
f(y)-f(x) \leq f_{1}(y)-f_{1}(x), \quad f(x)-f(y) \leq f_{1}(y)-f_{1}(x) .
$$

It is clear that these relations hold if and only if, for $x<y$,

$$
|f(y)-f(x)| \leq f_{1}(y)-f_{1}(x) .
$$

Remark 2.3. If $f$ is a Lipschitzian function defined on $\mathbb{R}$, that is, there exists a positive constant $C$ such that

$$
|f(x)-f(y)| \leq C|x-y|
$$

then

$$
f \ll \tilde{f}, \quad \text { with } \tilde{f}(x)=C x
$$

In general, if $f$ is a Lipschitzian function defined on $\mathbb{R}^{n}$, then $f \ll \tilde{f}$, where

$$
\tilde{f}\left(x_{1}, \ldots, x_{n}\right)=\operatorname{Lip}(f) \sum_{i=1}^{n} x_{i}, \quad \operatorname{Lip}(f)=\sup _{x \neq y} \frac{\left|f\left(x_{1}, \ldots, x_{n}\right)-f\left(y_{1}, \ldots, y_{n}\right)\right|}{\sum_{i=1}^{n}\left|x_{i}-y_{i}\right|}<\infty .
$$

Let $\left\{X_{n}, n \geq 1\right\}$ be a sequence of associated random variables. Let

$$
\begin{aligned}
& \text { (i) } Y_{n}=f_{n}\left(X_{1}, X_{2}, \ldots\right), \\
& \text { (ii) } \tilde{Y}_{n}=\tilde{f}_{n}\left(X_{1}, X_{2}, \ldots\right), \\
& \text { (iii) } f_{n} \ll \tilde{f}_{n}, \\
& \text { (iv) } E\left(Y_{n}^{2}\right)<\infty, \quad E\left(\tilde{Y}_{n}^{2}\right)<\infty, \quad \text { for } n \in \mathbb{N} \text {. }
\end{aligned}
$$


For convenience, we write that $Y_{n} \ll \widetilde{Y}_{n}$ if the conditions stated in (i)-(iv) hold. The functions $f_{n}, \tilde{f}_{n}$ are assumed to be real-valued and depend only on a finite number of $X_{n}$ 's. Let $S_{n}=\sum_{k=1}^{n} Y_{k}, \widetilde{S}_{n}=\sum_{k=1}^{n} \tilde{Y}_{k}$. Matula [8] proved the following result which will be useful in proving our results. He used them to prove the strong law of large numbers and the central limit theorem for nonmonotonic functions of associated random variables.

Lemma 2.4. Suppose the conditions stated above in (2.6) hold. Then

(i) $\operatorname{Var}\left(f_{n}\right) \leq \operatorname{Var}\left(\tilde{f}_{n}\right)$,

(ii) $\left|\operatorname{Cov}\left(f_{n}, \tilde{f}_{n}\right)\right| \leq \operatorname{Var}\left(\tilde{f}_{n}\right)$,

(iii) $\operatorname{Var}\left(S_{n}\right) \leq \operatorname{Var}\left(\widetilde{S}_{n}\right)$,

(iv) $f_{1}+f_{2}+\cdots+f_{n} \ll \tilde{f}_{1}+\tilde{f}_{2}+\cdots+\tilde{f}_{n}$,

(v) $\operatorname{Cov}\left(f_{1}+\tilde{f}_{1}, f_{2}+\tilde{f}_{2}\right) \leq 4 \operatorname{Cov}\left(\tilde{f}_{1}, \tilde{f}_{2}\right)$,

(vi) $\operatorname{Cov}\left(\tilde{f}_{1}-f_{1}, \tilde{f}_{2}-f_{2}\right) \leq 4 \operatorname{Cov}\left(\tilde{f}_{1}, \tilde{f}_{2}\right)$.

For completeness, now state the inequalities due to Newman and Wright [12] and Prakasa Rao [13] for associated random variables.

Lemma 2.5 (Newman and Wright). Suppose $X_{1}, X_{2}, \ldots, X_{m}$ are associated, mean zero, finite variance random variables, and $M_{m}^{*}=\max \left(S_{1}^{*}, S_{2}^{*}, \ldots, S_{m}^{*}\right)$, where $S_{n}^{*}=\sum_{i=1}^{n} X_{i}$. Then

$$
E\left(\left(M_{m}^{*}\right)^{2}\right) \leq \operatorname{Var}\left(S_{m}^{*}\right)
$$

Remark 2.6. Note that if $X_{1}, X_{2}, \ldots, X_{m}$ are associated random variables, then $-X_{1},-X_{2}$, $\ldots,-X_{m}$ also form a set of associated random variables. Let $M_{m}^{* *}=\max \left(-S_{1}^{*},-S_{2}^{*}, \ldots\right.$, $\left.-S_{m}^{*}\right)$ and $\widetilde{M}_{m}^{*}=\max \left(\left|S_{1}^{*}\right|,\left|S_{2}^{*}\right|, \ldots,\left|S_{m}^{*}\right|\right)$. Then $\widetilde{M}_{m}^{*}=\max \left(M_{m}^{*}, M_{m}^{* *}\right)$ and $\left(\widetilde{M}_{m}^{*}\right)^{2} \leq\left(M_{m}^{*}\right)^{2}$ $+\left(M_{m}^{* *}\right)^{2}$ so that

$$
E\left(\left(\widetilde{M}_{m}^{*}\right)^{2}\right) \leq 2 \operatorname{Var}\left(S_{m}^{*}\right)
$$

LEMma 2.7 (Prakasa Rao). Let $\left\{X_{n}, n \geq 1\right\}$ be an associated sequence of random variables with $\operatorname{Var}\left(X_{n}\right)=\sigma_{n}^{2}<\infty, n \geq 1$, and $\left\{b_{n}, n \geq 1\right\}$ a positive nondecreasing sequence of real numbers. Then, for any $\epsilon>0$,

$$
P\left(\max _{1 \leq k \leq n}\left|\frac{1}{b_{n}} \sum_{i=1}^{k}\left(X_{i}-E\left(X_{i}\right)\right)\right| \geq \epsilon\right) \leq \frac{4}{\epsilon^{2}}\left[\sum_{j=1}^{n} \frac{\operatorname{Var}\left(X_{j}\right)}{b_{j}^{2}}+\sum_{1 \leq j \neq k \leq n} \frac{\operatorname{Cov}\left(X_{j}, X_{k}\right)}{b_{j} b_{k}}\right] .
$$

\section{Main results}

We now extend the Newman and Wright's [12] result to nonmonotonic functions of associated random variables satisfying conditions (2.6). 
Theorem 3.1. Let $Y_{1}, Y_{2}, \ldots, Y_{m}$ be as defined in (2.6) with zero-mean and finite variances. Let $M_{m}=\max \left(\left|S_{1}\right|,\left|S_{2}\right|, \ldots,\left|S_{m}\right|\right)$. Then

$$
E\left(M_{m}^{2}\right) \leq(20) \operatorname{Var}\left(\tilde{S}_{m}\right)
$$

Proof. Observe that

$$
\begin{aligned}
\max _{1 \leq k \leq m}\left|S_{k}\right| & =\max _{1 \leq k \leq m}\left|\tilde{S}_{k}-S_{k}-E\left(\tilde{S}_{k}\right)-\tilde{S}_{k}+E\left(\tilde{S}_{k}\right)\right| \\
& \leq \max _{1 \leq k \leq m}\left|\tilde{S}_{k}-S_{k}-E\left(\tilde{S}_{k}\right)\right|+\max _{1 \leq k \leq m}\left|\tilde{S}_{k}-E\left(\tilde{S}_{k}\right)\right| .
\end{aligned}
$$

Note that $\widetilde{S}_{k}-E\left(\tilde{S}_{k}\right)$ and $\tilde{S}_{k}-S_{k}-E\left(\tilde{S}_{k}\right)$ are partial sums of associated random variables each with mean zero. Hence using the results of Newman and Wright [12], we get that

$$
\begin{aligned}
E\left(M_{m}^{2}\right) & \leq E\left(\max _{1 \leq k \leq m}\left|S_{k}\right|\right)^{2} \\
& \leq 2\left[E\left(\max _{1 \leq k \leq m}\left|\tilde{S}_{k}-S_{k}-E\left(\tilde{S}_{k}\right)\right|\right)^{2}+E\left(\max _{1 \leq k \leq m}\left|\tilde{S}_{k}-E\left(\tilde{S}_{k}\right)\right|\right)^{2}\right] \\
& \leq 4\left[\operatorname{Var}\left(\tilde{S}_{m}-S_{m}\right)+\operatorname{Var}\left(\tilde{S}_{m}\right)\right] \quad(\text { by Remark 2.6) } \\
& \leq 4\left[\operatorname{Var}\left(2 \tilde{S}_{m}\right)+\operatorname{Var}\left(\tilde{S}_{m}\right)\right]=20 \operatorname{Var}\left(\tilde{S}_{m}\right) .
\end{aligned}
$$

We have used the fact that

$$
\begin{aligned}
\operatorname{Var}\left(2 \tilde{S}_{n}\right) & =\operatorname{Var}\left(\widetilde{S}_{n}-S_{n}+\widetilde{S}_{n}+S_{n}\right) \\
& =\operatorname{Var}\left(\tilde{S}_{n}-S_{n}\right)+\operatorname{Var}\left(\tilde{S}_{n}+S_{n}\right)+2 \operatorname{Cov}\left(\tilde{S}_{n}+S_{n}, \widetilde{S}_{n}-S_{n}\right) .
\end{aligned}
$$

Since $\widetilde{S}_{n}+S_{n}$ and $\widetilde{S}_{n}-S_{n}$ are nondecreasing functions of associated random variables, it follows that $\operatorname{Cov}\left(\widetilde{S}_{n}+S_{n}, \widetilde{S}_{n}-S_{n}\right) \geq 0$. Hence $\operatorname{Var}\left(2 \widetilde{S}_{n}\right) \geq \operatorname{Var}\left(\widetilde{S}_{n}-S_{n}\right)$.

We now prove a Hajek-Renyi-type inequality for some nonmonotonic functions of associated random variables satisfying conditions (2.6).

Theorem 3.2. Let $\left\{Y_{n}, n \geq 1\right\}$ be sequence of nonmonotonic functions of associated random variables as defined in (2.6). Suppose that $Y_{n} \ll \tilde{Y}_{n}, n \geq 1$. Let $\left\{b_{n}, n \geq 1\right\}$ be a positive nondecreasing sequence of real numbers. Then for any $\epsilon>0$,

$$
P\left(\max _{1 \leq k \leq n}\left|\frac{1}{b_{n}} \sum_{i=1}^{k}\left(Y_{i}-E\left(Y_{i}\right)\right)\right| \geq \epsilon\right) \leq(80) \epsilon^{-2}\left[\sum_{j=1}^{n} \frac{\operatorname{Var}\left(\tilde{Y}_{j}\right)}{b_{j}^{2}}+\sum_{1 \leq j \neq k \leq n} \frac{\operatorname{Cov}\left(\tilde{Y}_{j}, \tilde{Y}_{k}\right)}{b_{j} b_{k}}\right] .
$$


Proof. Let $T_{n}=\sum_{j=1}^{n}\left(Y_{j}-E\left(Y_{j}\right)\right)$. Note that

$$
\begin{aligned}
P\left[\max _{1 \leq k \leq n}\left|\frac{T_{k}}{b_{k}}\right| \geq \epsilon\right] & \\
= & P\left[\max _{1 \leq k \leq n}\left|\frac{\left|\widetilde{T}_{k}-T_{k}-E\left(\tilde{T}_{k}\right)-\widetilde{T}_{k}+E\left(\tilde{T}_{k}\right)\right|}{b_{k}}\right| \geq \epsilon\right] \\
\leq & P\left[\max _{1 \leq k \leq n}\left|\frac{\tilde{T}_{k}-T_{k}-E\left(\tilde{T}_{k}\right)}{b_{k}}\right| \geq \frac{\epsilon}{2}\right]+P\left[\max _{1 \leq k \leq n}\left|\frac{\left|\widetilde{T}_{k}-E\left(\tilde{T}_{k}\right)\right|}{b_{k}}\right| \geq \frac{\epsilon}{2}\right] \\
\leq & (16) \epsilon^{-2}\left[\sum_{j=1}^{n} \frac{\operatorname{Var}\left(\tilde{Y}_{j}-Y_{j}\right)}{b_{j}^{2}}+\sum_{1 \leq j \neq k \leq n} \frac{\operatorname{Cov}\left(\tilde{Y}_{j}-Y_{j}, \tilde{Y}_{k}-Y_{k}\right)}{b_{j} b_{k}}\right] \\
& +(16) \epsilon^{-2}\left[\sum_{j=1}^{n} \frac{\operatorname{Var}\left(\tilde{Y}_{j}\right)}{b_{j}^{2}}+\sum_{1 \leq j \neq k \leq n} \frac{\operatorname{Cov}\left(\tilde{Y}_{j}, \tilde{Y}_{k}\right)}{b_{j} b_{k}}\right] .
\end{aligned}
$$

The result follows by applying the following inequalities:

$$
\operatorname{Var}\left(\tilde{Y}_{j}-Y_{j}\right) \leq 4 \operatorname{Var}\left(\tilde{Y}_{j}\right), \quad \operatorname{Cov}\left(\tilde{Y}_{j}-Y_{j}, \tilde{Y}_{k}-Y_{k}\right) \leq 4 \operatorname{Cov}\left(\tilde{Y}_{j}, \tilde{Y}_{k}\right)
$$

\section{Applications}

Let $C$ denote a generic positive constant.

Corollary 4.1. Let $\left\{Y_{n}, n \geq 1\right\}$ be sequence of nonmonotonic functions of associated random variables satisfying the conditions in (2.6). Assume that

$$
\sum_{j=1}^{\infty} \operatorname{Var}\left(\tilde{Y}_{j}\right)+\sum_{1 \leq j \neq k<\infty} \operatorname{Cov}\left(\tilde{Y}_{j}, \tilde{Y}_{k}\right)<\infty .
$$

Then $\sum_{j=1}^{\infty}\left(Y_{j}-E Y_{j}\right)$ converges almost surely.

Proof. Without loss of generality, assume that $E Y_{j}=0$ for all $j \geq 1$. Let $T_{n}=\sum_{j=1}^{n} Y_{j}$ and $\epsilon>0$. Using Theorem 3.2 is easy to see that

$$
\begin{aligned}
& P\left(\sup _{k, m \geq n}\left|T_{k}-T_{m}\right| \geq \epsilon\right) \\
& \quad \leq P\left(\sup _{k \geq n}\left|T_{k}-T_{n}\right| \geq \frac{\epsilon}{2}\right)+P\left(\sup _{m \geq n}\left|T_{m}-T_{n}\right| \geq \frac{\epsilon}{2}\right) \\
& \quad \leq C \limsup _{\mathbb{N} \rightarrow \infty} P\left(\sup _{n \leq k \leq \mathbb{N}}\left|T_{k}-T_{n}\right| \geq \frac{\epsilon}{2}\right) \\
& \quad \leq C \epsilon^{-2}\left[\sum_{j=n}^{\infty} \operatorname{Var}\left(\tilde{Y}_{j}\right)+\sum_{n \leq j \neq k<\infty} \operatorname{Cov}\left(\tilde{Y}_{j}, \tilde{Y}_{k}\right)\right] .
\end{aligned}
$$


The last term tends to zero as $n \rightarrow \infty$ because of (4.1). Hence the sequence of random variables $\left\{T_{n}, n \geq 1\right\}$ is Cauchy almost surely which implies that $T_{n}$ converges almost surely.

The following corollary proves the strong law of large numbers for nonmonotonic functions of associated random variables.

Corollary 4.2. Let $\left\{Y_{n}, n \geq 1\right\}$ be sequence of nonmonotonic functions of associated random variables satisfying the conditions in (2.6). Suppose that

$$
\sum_{j=1}^{\infty} \frac{\operatorname{Var}\left(\tilde{Y}_{j}\right)}{b_{j}^{2}}+\sum_{1 \leq j \neq k<\infty} \frac{\operatorname{Cov}\left(\tilde{Y}_{j}, \tilde{Y}_{k}\right)}{b_{j} b_{k}}<\infty .
$$

Then $\left(1 / b_{n}\right) \sum_{j=1}^{n}\left(Y_{j}-E Y_{j}\right)$ converges to zero almost surely as $n \rightarrow \infty$.

Proof. The proof is an immediate consequence of Theorem 3.2 and the Kronecker lemma (Chung [3]).

Remark 4.3. Birkel [2] proved a strong law of large numbers for positively dependent random variables. Prakasa Rao [13] proved a strong law of large numbers for associated sequences as a consequence of the Hajek-Renyi-type inequality. Marcinkiewicz-Zygmundtype strong law of large numbers for associated random variables, for which the second moment is not necessarily finite, was studied in Louhichi [7]. Strong law of large numbers for monotone functions of associated sequences follows from these results since monotone functions of associated sequences are associated. However Corollary 4.2 gives sufficient conditions for the strong law of large numbers to hold for possibly nonmonotonic functions of associated sequences whose second moments are finite.

For any random variable $X$ and any constant $k>0$, define $X^{k}=X$ if $|X| \leq k, X^{k}=-k$ if $X<-k$, and $X^{k}=k$ if $X>k$. The following theorem is an analogue of the three series theorem for nonmonotonic functions of associated random variables.

Corollary 4.4. Let $\left\{Y_{n}, n \geq 1\right\}$ be sequence of nonmonotonic functions of associated random variables. Further suppose that there exists a constant $k>0$ such that $Y_{n}^{k} \ll \tilde{Y}_{n}^{k}$ satisfying the conditions in (2.6) and

$$
\begin{aligned}
& \sum_{n=1}^{\infty} P\left[\left|Y_{n}\right| \geq k\right]<\infty, \quad \sum_{n=1}^{\infty} E\left(Y_{n}^{k}\right)<\infty, \\
& \sum_{j=1}^{\infty} \operatorname{Var}\left(\tilde{Y}_{j}^{k}\right)+\sum_{1 \leq j \neq j^{\prime}<\infty} \operatorname{Cov}\left(\tilde{Y}_{j}^{k}, \tilde{Y}_{j^{\prime}}^{k}\right)<\infty .
\end{aligned}
$$

Then $\sum_{n=1}^{\infty} Y_{n}$ converges almost surely. 
Corollary 4.5. Let $\left\{Y_{n}, n \geq 1\right\}$ be sequence of nonmonotonic functions of associated random variables satisfying the conditions in (2.6). Suppose

$$
\sum_{j=1}^{\infty} \frac{\operatorname{Var}\left(\tilde{Y}_{j}\right)}{b_{j}^{2}}+\sum_{1 \leq j \neq k<\infty} \frac{\operatorname{Cov}\left(\tilde{Y}_{j}, \tilde{Y}_{k}\right)}{b_{j} b_{k}}<\infty .
$$

Let $T_{n}=\sum_{j=1}^{n}\left(Y_{j}-E\left(Y_{j}\right)\right)$. Then, for any $0<r<2$,

$$
E\left[\sup _{n}\left(\frac{\left|T_{n}\right|}{b_{n}}\right)^{r}\right]<\infty \text {. }
$$

Proof. Note that

$$
E\left[\sup _{n}\left(\frac{\left|T_{n}\right|}{b_{n}}\right)^{r}\right]<\infty
$$

if and only if

$$
\int_{1}^{\infty} P\left(\sup _{n}\left(\frac{\left|T_{n}\right|}{b_{n}}\right)^{r}>t^{1 / r}\right) d t<\infty .
$$

The last inequality holds because of Theorem 3.2 and condition (4.5). Hence the result stated in (4.6) holds.

\section{Acknowledgment}

The authors thank the referee for the comments and suggestions which have led to an improved presentation.

\section{References}

[1] R. E. Barlow and F. Proschan, Statistical Theory of Reliability and Life Testing: Probability Models, Holt, Rinehart and Winston, New York, 1981.

[2] T. Birkel, A note on the strong law of large numbers for positively dependent random variables, Statistics \& Probability Letters 7 (1988), no. 1, 17-20.

[3] K. L. Chung, A Course in Probability Theory, Academic Press, New York, 1974.

[4] J. T. Cox and G. Grimmett, Central limit theorems for associated random variables and the percolation model, The Annals of Probability 12 (1984), no. 2, 514-528.

[5] I. Dewan and B. L. S. Prakasa Rao, Asymptotic normality for U-statistics of associated random variables, Journal of Statistical Planning and Inference 97 (2001), no. 2, 201-225.

[6] J. Esary, F. Proschan, and D. Walkup, Association of random variables, with applications, Annals of Mathematical Statistics 38 (1967), 1466-1474.

[7] S. Louhichi, Convergence rates in the strong law for associated random variables, Probability and Mathematical Statistics 20 (2000), no. 1, 203-214.

[8] P. Matula, Limit theorems for sums of nonmonotonic functions of associated random variables, Journal of Mathematical Sciences 105 (2001), no. 6, 2590-2593.

[9] C. M. Newman, Normal fluctuations and the FKG inequalities, Communications in Mathematical Physics 74 (1980), no. 2, 119-128.

[10] A general central limit theorem for FKG systems, Communications in Mathematical Physics 91 (1983), no. 1, 75-80. 
[11] _ Asymptotic independence and limit theorems for positively and negatively dependent random variables, Inequalities in Statistics and Probability (Lincoln, Neb, 1982) (Y. L. Tong, ed.), vol. 5, Institute of Mathematical Statistics, California, 1984, pp. 127-140.

[12] C. M. Newman and A. L. Wright, An invariance principle for certain dependent sequences, The Annals of Probability 9 (1981), no. 4, 671-675.

[13] B. L. S. Prakasa Rao, Hajek-Renyi-type inequality for associated sequences, Statistics \& Probability Letters 57 (2002), no. 2, 139-143.

[14] G. G. Roussas, Positive and negative dependence with some statistical applications, Asymptotics, Nonparametrics, and Time Series (S. Ghosh, ed.), vol. 158, Marcel Dekker, New York, 1999, pp. 757-788.

Isha Dewan: Theoretical Statistics and Mathematics Unit, Indian Statistical Institute,

New Delhi 110016, India

E-mail address: isha@isid2.isid.ac.in

B. L. S. Prakasa Rao: Department of Mathematics and Statistics, University of Hyderabad, Hyderabad 500046, India

E-mail address: blsprsm@uohyd.ernet.in 